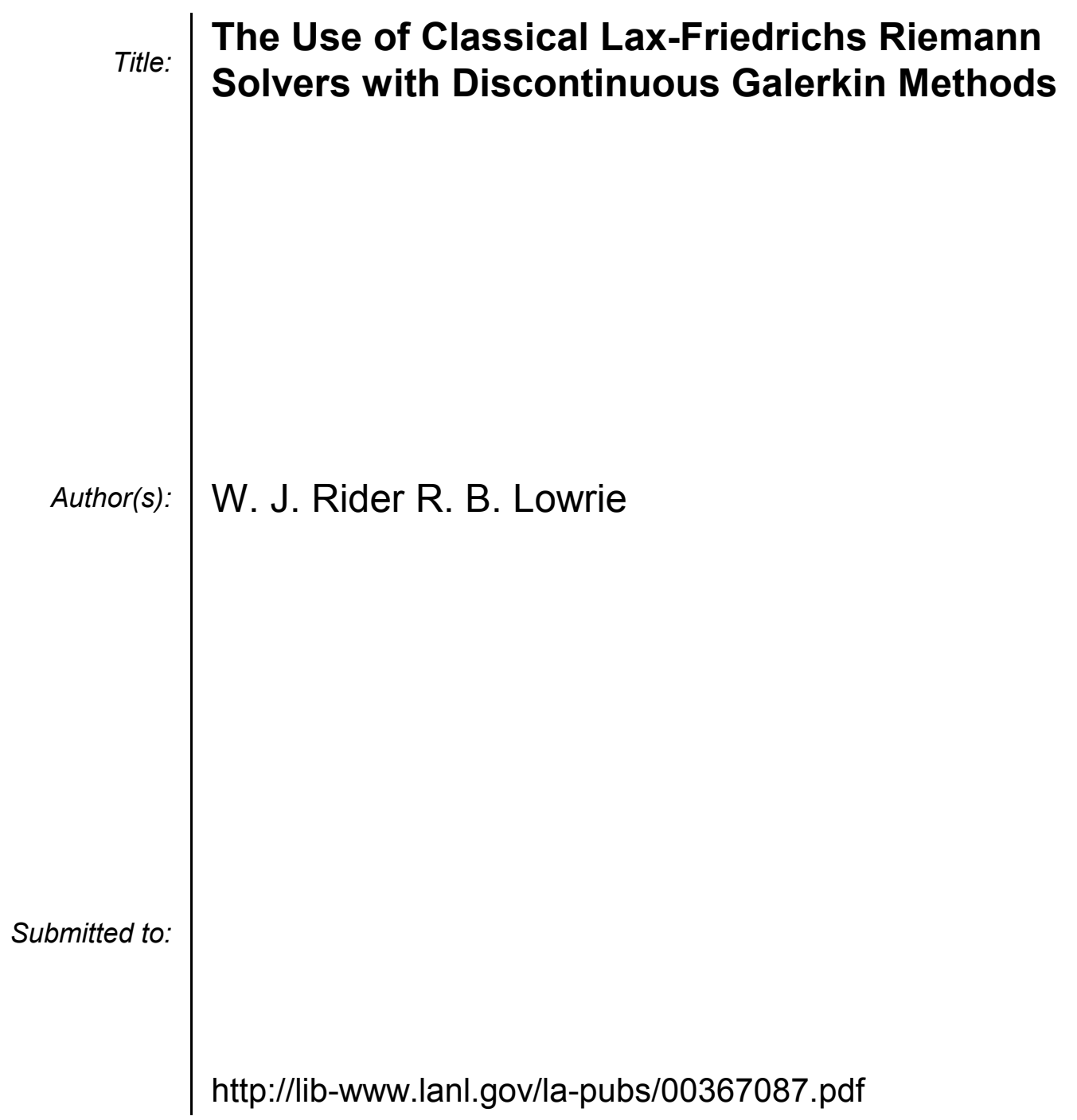

Los Alamos National Laboratory, an affirmative action/equal opportunity employer, is operated by the University of California for the U.S. Department of Energy under contract W-7405-ENG-36. By acceptance of this article, the publisher recognizes that the U.S. Government retains a nonexclusive, royaltyfree license to publish or reproduce the published form of this contribution, or to allow others to do so, for U.S. Government purposes. Los Alamos National Laboratory requests that the publisher identify this article as work performed under the auspices of the U.S. Department of Energy. Los Alamos National Laboratory strongly supports academic freedom and a researcher's right to publish; as an institution, however, the Laboratory does not endorse the viewpoint of a publication or guarantee its technical correctness. 


\title{
The Use of Classical Lax-Friedrichs Riemann Solvers with Discontinuous Galerkin Methods
}

\author{
W. J. Rider \\ R. B. Lowrie \\ Los Alamos National Laboratory, MS D413, Los Alamos, NM 87545
}

\begin{abstract}
While conducting a von Neumann stability analysis of discontinuous Galerkin methods we found that the standard Lax-Friedrichs $(\mathrm{LxF})$ Riemann solver is unstable for all time-step sizes. A simple modification of the Riemann solver's dissipation returns the method to stability. Furthermore, the method has a smaller truncation error than the corresponding method with an upwind flux for the RK2-DG(1) method. These results are confirmed upon testing.
\end{abstract}

\section{Introduction}

Lax-Friedrichs Riemann solvers are commonly used to enhance the robustness of Godunovtype methods [7]. Here we present numerical stability analysis and computations that demonstrate some subtle aspects to the use of LxF Riemann solvers with Discontinuous Galerkin (DG) methods. The simplicity and economy of the resulting methods motivate us. Used blindly in one of its classical forms, the method is linearly unstable, but can be modified to recover numerical stability. Moreover, the modified method has a smaller truncation error than its upwind counterpart.

We have conducted a set of numerical stability analyses covering a variety of time integration schemes ranging from fully discrete to semi-discrete forms. We followed the earlier analysis techniques of Lowrie [3]. While the analysis recovered the expected results for a variety of methods using classical upwinding as a Riemann solver, the use of the standard Lax-Friedrichs Riemann solver produced a linearly unstable method (see Section 2). This was confirmed upon application of the method to a simple wave equation and subsequently on Burgers' equation in Section 3.

Combining Godunov methods and finite element methods is an attractive approach for solving hyperbolic conservation laws. Such a combination is found with discontinuous Galerkin methods where a discontinuous basis is used in the Galerkin approximation. This method is rather natural for approximating weak solutions. As a necessity for resolving the discontinuities at element boundaries Riemann solvers are employed to determine a unique inter-element flux. In addition, DG methods are more compact than typical high resolution Godunov methods not requiring extensive memory accesses to surrounding cells or elements. For the purpose of nonlinear limiting and flux evaluations only nearest neighbors are required to be accessed.

Discontinuous Galerkin methods were first introduced by Reed and Hill [5] for neutron transport. Subsequently the method has found far greater use in the hydrodynamics community although Morel and coworkers have revitalized its use in radiation transport [4]. Key developments were made by Cockburn and Shu (see [1] for example). Recent work has culminated in a robust, high-resolution method for conservation laws [2]. 
Generically we are interested in solving a conservation law, $u_{t}+(f(u))_{x}=0$. In its simplest form the finite difference equation for updating the conservation law is

$$
\frac{\partial u_{j}}{\partial t}=-\frac{1}{\Delta x}\left[f\left(u_{j+\frac{1}{2}}\right)-f\left(u_{j-\frac{1}{2}}\right)\right],
$$

where we have explicitly employed a method of lines form. One can write the linear basis for a DG(1) scheme as $u_{j}+s_{j}\left(x-x_{j}\right), x \in\left[x_{j}-\Delta x / 2, x_{j}+\Delta x / 2\right]$ For DG(1) the slope, $s_{j}$ is updated using the following form,

$$
\frac{\partial s_{j}}{\partial t}=-\frac{6}{\Delta x^{2}}\left[f\left(u_{j-\frac{1}{2}}\right)+f\left(u_{j+\frac{1}{2}}\right)\right]+\frac{12}{\Delta x^{2}} \int_{-\Delta x / 2}^{\Delta x / 2} f(u) d x .
$$

The DG(2) scheme uses a quadratic basis $u_{j}+s_{j}\left(x-x_{j}\right)+q_{j}\left[\left(x-x_{j}\right)^{2}-\Delta x^{2} / 12\right], x \in$ $\left[x_{j}-\Delta x / 2, x_{j}+\Delta x / 2\right]$ The form for updating $s_{j}$ is retained and the quadratic term, $q_{j}$, is updated using the following equation,

$$
\frac{\partial q_{j}}{\partial t}=-\frac{30}{\Delta x^{3}}\left[f\left(u_{j+\frac{1}{2}}\right)-f\left(u_{j-\frac{1}{2}}\right)\right]+\frac{360}{\Delta x^{3}} \int_{-\Delta x / 2}^{\Delta x / 2} f(u)\left(x-x_{o}\right) d x .
$$

We use these discrete forms in conducting our stability analysis in Section 2.

We will integrate these methods with Runge-Kutta methods based on a TVD principle [6]. Typically, we will use a second-order integrator with DG(1). This method for $u_{t}=F(u)$ which is equivalent to Heun's method is $u^{n+1}=\frac{1}{2}\left(u^{n}+u^{1}+\Delta t F\left(u^{1}\right)\right) ; u^{1}=u^{n}+\Delta t F\left(u^{n}\right)$. Wth DG(1) this method will be referred to a RK2-DG(1), and we expect second-order accuracy for sufficiently smooth flows. With the DG(2) method we will use a third-order integrator, $u^{n+1}=\frac{1}{3}\left(u^{n}+2 u^{2}+2 \Delta t F\left(u^{2}\right)\right) ; u^{2}=\frac{1}{4}\left(3 u^{n}+u^{1}+\Delta t F\left(u^{1}\right)\right) u^{1}=u^{n}+\Delta t F\left(u^{n}\right)$. With DG(2) this is the RK3-DG(2) method. We expect third-order accuracy with this method. Next, we discuss Riemann solvers, their formulation and implications for Godunovtype methods.

In Godunov-type methods, Riemann solvers play an essential role. Because the quest for correct physical solutions depends crucially on satisfying an entropy condition, sufficiently dissipative Riemann solutions are important. Exact Riemann solvers (upwind in the scalar case) are only marginally entropy satisfying. Indeed it defines a bounding case. More dissipation can be entertained to the limit of a Lax-Friedrichs method. It is also important to note that the Lax-Friedrichs method is as dissipative as a stable explicit method can be for basic methods.

The importance of the dissipative Riemann solvers is in the design of robust numerical methods. In difficult circumstance one can use more dissipation via the LxF Riemann solvers to achieve robustness. This combined with nonlinear spatial differencing provides a reliable numerical method for a variety of problems including some of the most challenging in existence. The Godunov flux is the least dissipative flux that satisfies an entropy condition (an E-flux, $\left.\left(f\left(u_{l}, u_{r}\right)-f(u)\right)\left(u_{r}-u_{l}\right) \leq 0, u \in\left[u_{l}, u_{r}\right]\right)$, and for typical schemes LxF is the most dissipative flux that leads to a stable scheme.

In the case of a scalar equation, the basic upwind numerical flux is

$$
f\left(u_{l}, u_{r}\right)=\frac{1}{2}\left(f\left(u_{l}\right)+f\left(u_{r}\right)\right)-\frac{\left|f^{\prime}(u)\right|}{2}\left(u_{r}-u_{l}\right),
$$

where $f^{\prime}(u)$ is the characteristic velocity at the interface. The classical LxF flux changes the dissipation to an upper bound, $\Delta x / \Delta t$,

$$
f\left(u_{l}, u_{r}\right)=\frac{1}{2}\left(f\left(u_{l}\right)+f\left(u_{r}\right)\right)-\frac{\Delta x}{2 \Delta t}\left(u_{r}-u_{l}\right) .
$$


Cockburn and Shu [2] usually use a local Lax-Friedrichs (LLxF) method rather than LxF. In LLxF, the dissipation magnitude in (1.4), $\left|f^{\prime}(u)\right|=\max \left|f^{\prime}(u)\right|$. The dissipation in (1.5) is an upper bound for the dissipation.

Each of these schemes can be extended to systems of nonlinear conservation laws. For Burgers' equation the Godunov flux is used in place of $(1.4), f\left(u_{l}, u_{r}\right)=$ $\frac{1}{2} \max \left[\max \left(0, u_{l}\right)^{2}, \min \left(0, u_{r}\right)^{2}\right]$. The importance of $\mathrm{LxF}$ to the construction of robust numerical schemes cannot be overstated. LxF provides the ability to securely produce adequate entropy through the numerical flux. In the next section, we discuss the stability analysis of DG schemes with the Riemann solvers discussed above.

\section{Stability Analysis}

In order to investigate the stability of different DG implementations we conducted at stability analysis using Mathematica and Maple. The analysis follows the form and philosophy detailed by Lowrie [3]. We develop the method as a finite difference method where more than one degree of freedom is evolved per mesh cell. For a DG(1) method we have the cell average and its moment (or linear expansion coefficient/slope), for DG(2) we have a second moment (or parabolic expansion coefficient). For a scalar wave equation this changes the stability analysis to a matrix analysis problem. Aside from the matrix analysis details the analysis is conducted as any other von Neumann stability analysis.

In keeping with this, we describe the update of the variables as $U_{j}^{n+1}(\xi)=$ $g U_{j}^{n}(\xi) ; U_{j}^{n}(\xi)=e^{2 \theta(j+\xi)}$. where $U_{j}=\left(u_{j}, s_{j}, q_{j}\right)^{T}$. We also have the exact evolution for

$u_{t}+a u_{x}=0, U_{j}^{n+1}(\xi)=g_{\text {exact }} u_{j}^{n}(\xi) ; g_{\text {exact }}=e^{\imath \nu \theta}$. where $\nu=a \Delta t / \Delta x$ is the Courant number. Because we have more than one unknown per mesh cell, we will have several eigenvalues describing the stability, $1 \leq\left|g_{1}\right| \geq\left|g_{2}\right|$ for $\mathrm{DG}(1)$ and $1 \leq\left|g_{1}\right| \geq\left|g_{2}\right| \geq\left|g_{3}\right|$. for DG(2). The first eigenvalue describes the accuracy of the solution which can be estimated through $g_{1}-g_{\text {exact }}$. We can also describe the amplitude and phase error of the method through expansions of $g_{1}$.

The difficult aspect of analysis for DG methods is recognizing the accurate and spurious modes for the method. One mode is the accurate mode where we wish to examine the accuracy of the method. For stability the spurious of non-physical modes must be damped more greatly than the accurate mode. The spurious mode manifests itself most acutely in what van Leer refers to as "stegasaur bias" from his 1977 paper [8].

We discovered that the simple modification of the time step used in the mesh spacing to time step size ratio in the Riemann solver recovers linear stability. This modification consists of multiplying this ratio by the stability limit for the method. For example, a DG(1) method with a second-order TVD Runge-Kutta (RK) method and the upwind Riemann solver has an explicit stability limit of $1 / 3(1 / 5$ for RK3) [1]. When we replace the upwind flux with the standard LxF flux the method is unconditionally unstable. The same results holds for DG(2). We modify the LxF flux from to

$$
f\left(u_{l}, u_{r}\right)=\frac{1}{2}\left(f\left(u_{l}\right)+f\left(u_{r}\right)\right)-\frac{z \Delta x}{2 \Delta t}\left(u_{r}-u_{l}\right),
$$

if $z$ is set equal to the explicit stability limit for the scheme with the upwind flux, stability for the method is recovered over the original stability range. This will be denoted as the mLxF flux. In Figure 1. we show the relative damping $\left|g_{1}\right| /\left|g_{2}\right|$ of the two DG(1) schemes. The mLxF flux damps the spurious mode less than the upwind scheme.

Moreover, the truncation error reduces from the case of upwinding being equal to it at the stability limit, but reducing as the CFL number goes to zero. For RK2-DG(1) with an 

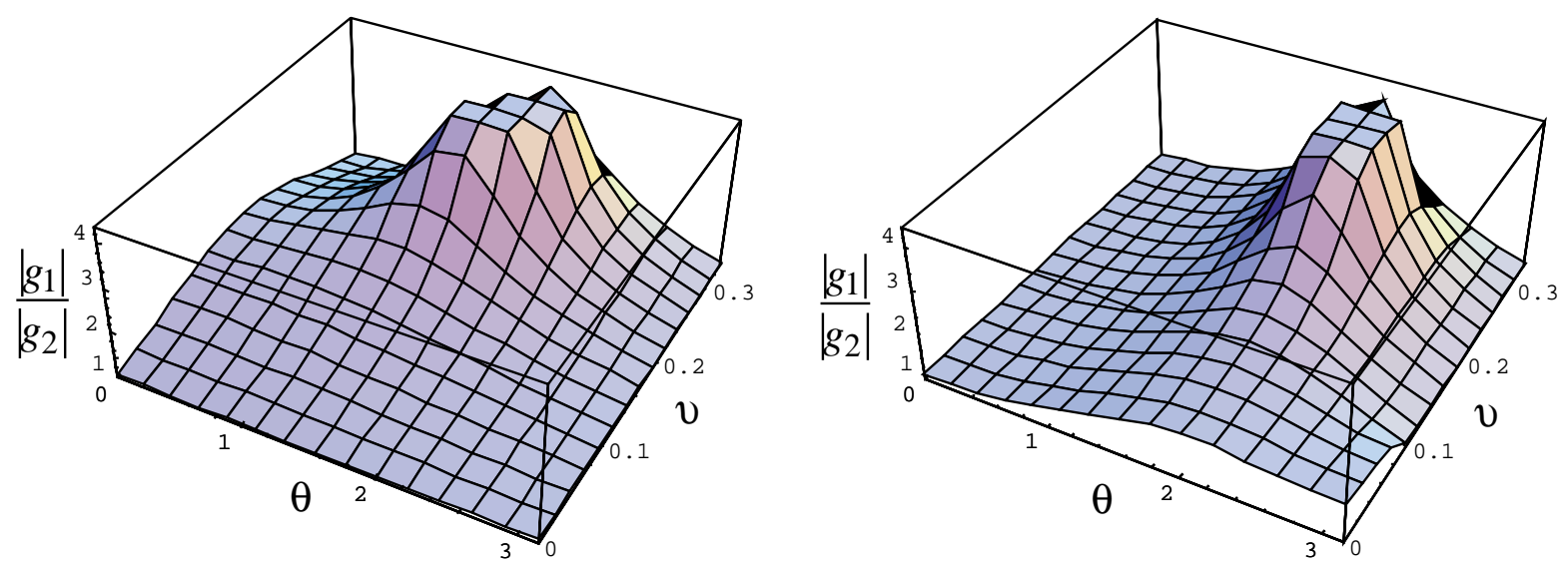

Figure 1: Comparing RK2 DG(1) with upwind and modified LxF Riemann solvers for the relative damping of the spurious mode compared with the accurate mode.

upwind flux the truncation error is $-\frac{\nu^{3}}{6} u_{x x x}-\frac{1}{72}\left(\nu+3 \nu^{4}\right) u_{x x x x}$. With the mLxF flux, the RK2-DG(1) truncation error is $-\frac{\nu^{3}}{6} u_{x x x}-\frac{1}{24}\left(\nu^{2}+\nu^{4}\right) u_{x x x x}$. The scheme with the mLxF flux has smaller truncation error for all stable time steps. For the RK3-DG(2) schemes the truncation error estimate is beyond the ability of the symbolic algebra software to compute!

\section{Testing the Stability Analysis}

While DG(1) more accurate especially as the Courant number becomes small, the performance on discontinuous waves appears to be unharmed as shown in Table 1 and Figure 2 The smaller error for the Gaussian pulse is evident while the performance on the step is similar. No limiting is used although their inclusion poses no particular difficulties. The results for RK3-DG(2) show slight differences. The mLxF flux no longer shows smaller errors, but rather is slightly larger.

Table 2 shows that the results found for the scalar wave equation are recovered for Burgers' equation. The RK2-DG(1) with mLxF produces significantly smaller errors than the upwind flux while the solution is smooth. The opposite is true for RK3-DG(2). In broad terms the order of the schemes is not impacted negatively by the mLxF flux. Figures 3 and 4 show the results for two times. The accentuation of the spurious mode by the mLxF flux is obvious after the shock forms (at $t=1 / 2 \pi$ ). Here any oscillations are not localized at the shock because of the centered nature of $\mathrm{mLxF}$. The greater spurious mode amplification can explain this behavior. Nonlinear limiting removes much of this character from the solution. In both cases, the evolution of the mean values is not adversely effected.

We have found a stability problem with the classical Lax-Friedrichs numerical flux when used in conjunction with discontinuous Galerkin methods. Fortunately, this difficulty can be easily remedied through modifying the numerical flux function. The numerical behavior of the method can be used to explain more general behavior of results with methods between upwinding and LxF (i.e. LLxF). We also have the pleasant byproduct of reduced truncation error resulting from this modification of the method for RK2-DG(1). Additionally, we find that the new method does not have degraded performance on discontinuous waves and shocks. 

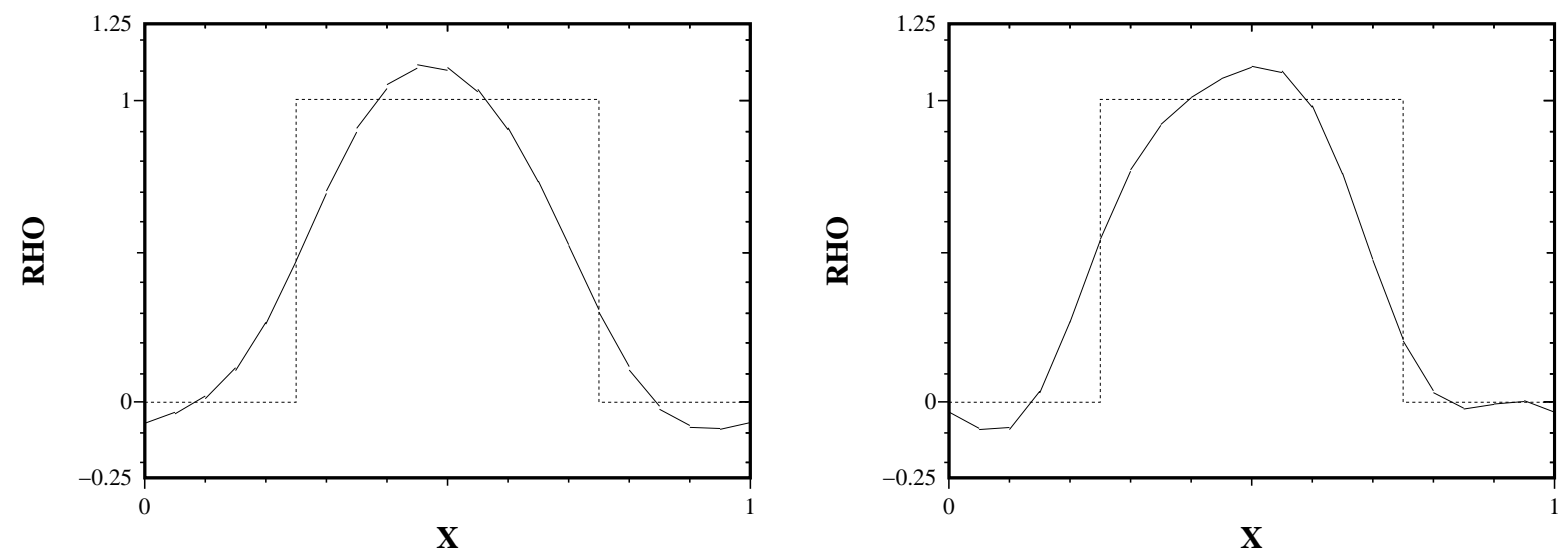

Figure 2: RK2-DG(1) results for a step function. On the left the results with the upwind flux is shown and the right shows the mLxF flux. and a square wave. The Courant number is 0.1 and the results with 20 cells is shown. for 10 periods around the grid. The solid line shows the reconstruction defined by the scheme and the dashed line shows the exact solution.

\begin{tabular}{|l|l|c|c|c|c|c|}
\hline \hline Scheme & Norm & Error 20 & Rate & Error 40 & Rate & Error 80 \\
\hline \hline RK2-DG(1) & $L_{1}$ & $3.97 \times 10^{-2}$ & 2.49 & $7.06 \times 10^{-3}$ & 2.73 & $1.06 \times 10^{-3}$ \\
Upwind & $L_{2}$ & $4.91 \times 10^{-2}$ & 2.39 & $9.36 \times 10^{-3}$ & 2.70 & $1.44 \times 10^{-3}$ \\
& $L_{\infty}$ & $1.04 \times 10^{-1}$ & 2.27 & $2.16 \times 10^{-2}$ & 2.69 & $3.35 \times 10^{-3}$ \\
\hline RK2-DG(1) & $L_{1}$ & $1.72 \times 10^{-2}$ & 2.77 & $2.53 \times 10^{-3}$ & 2.09 & $5.93 \times 10^{-4}$ \\
mLxF & $L_{2}$ & $2.22 \times 10^{-2}$ & 2.66 & $3.51 \times 10^{-3}$ & 2.13 & $8.01 \times 10^{-4}$ \\
& $L_{\infty}$ & $4.86 \times 10^{-2}$ & 2.68 & $7.56 \times 10^{-3}$ & 2.15 & $1.70 \times 10^{-3}$ \\
\hline RK3-DG(2) & $L_{1}$ & $5.47 \times 10^{-4}$ & 3.87 & $3.75 \times 10^{-5}$ & 2.91 & $4.99 \times 10^{-6}$ \\
Upwind & $L_{2}$ & $7.55 \times 10^{-4}$ & 3.95 & $4.89 \times 10^{-5}$ & 2.64 & $7.85 \times 10^{-6}$ \\
& $L_{\infty}$ & $1.76 \times 10^{-3}$ & 3.91 & $1.17 \times 10^{-4}$ & 1.93 & $3.06 \times 10^{-5}$ \\
\hline RK3-DG(2) & $L_{1}$ & $6.88 \times 10^{-4}$ & 3.92 & $4.54 \times 10^{-5}$ & 3.05 & $5.47 \times 10^{-6}$ \\
mLxF & $L_{2}$ & $9.28 \times 10^{-4}$ & 3.97 & $5.94 \times 10^{-5}$ & 2.78 & $8.63 \times 10^{-6}$ \\
& $L_{\infty}$ & $2.25 \times 10^{-3}$ & 3.97 & $1.44 \times 10^{-4}$ & 1.88 & $3.90 \times 10^{-5}$ \\
\hline \hline
\end{tabular}

Table 1: Error norms using RK2-DG(1) and RK3-DG(2) with upwind and mLxF Remann solvers on advecting a Gaussian for 10 periods. 


\begin{tabular}{|l|l|l|l|l|l|l|}
\hline \hline Scheme & Norm & Error 20-40 & Rate & Error 40-80 & Rate & Error 80-160 \\
\hline \hline RK2-DG(1) & $L_{1}$ & $8.62 \times 10^{-4}$ & 2.60 & $1.42 \times 10^{-4}$ & 2.66 & $2.25 \times 10^{-5}$ \\
Godunov & $L_{2}$ & $1.49 \times 10^{-3}$ & 2.20 & $3.25 \times 10^{-4}$ & 2.66 & $5.14 \times 10^{-5}$ \\
& $L_{\infty}$ & $4.07 \times 10^{-3}$ & 1.61 & $1.33 \times 10^{-3}$ & 2.44 & $2.45 \times 10^{-4}$ \\
\hline RK2-DG(1) & $L_{1}$ & $6.95 \times 10^{-4}$ & 3.39 & $6.64 \times 10^{-5}$ & 3.32 & $6.64 \times 10^{-6}$ \\
mLxF & $L_{2}$ & $1.89 \times 10^{-3}$ & 3.50 & $1.67 \times 10^{-4}$ & 3.89 & $1.13 \times 10^{-5}$ \\
& $L_{\infty}$ & $5.97 \times 10^{-3}$ & 3.09 & $7.01 \times 10^{-4}$ & 3.88 & $4.77 \times 10^{-5}$ \\
\hline RK3-DG(2) & $L_{1}$ & $4.76 \times 10^{-5}$ & 3.96 & $3.07 \times 10^{-6}$ & 3.05 & $3.71 \times 10^{-7}$ \\
Godunov & $L_{2}$ & $7.03 \times 10^{-5}$ & 3.79 & $5.08 \times 10^{-6}$ & 2.32 & $1.02 \times 10^{-6}$ \\
& $L_{\infty}$ & $1.57 \times 10^{-4}$ & 3.31 & $1.59 \times 10^{-5}$ & 1.61 & $5.21 \times 10^{-6}$ \\
\hline RK3-DG(2) & $L_{1}$ & $9.06 \times 10^{-5}$ & 3.15 & $1.02 \times 10^{-5}$ & 3.57 & $8.58 \times 10^{-7}$ \\
mLxF & $L_{2}$ & $1.74 \times 10^{-4}$ & 2.85 & $2.41 \times 10^{-5}$ & 3.36 & $2.35 \times 10^{-6}$ \\
& $L_{\infty}$ & $5.20 \times 10^{-4}$ & 2.42 & $9.67 \times 10^{-5}$ & 3.09 & $1.14 \times 10^{-5}$ \\
\hline \hline
\end{tabular}

Table 2: Error norms using RK2-DG(1) and RK3-DG(2) with upwind and mLxF Remann solvers on Burgers' equations at $\mathrm{t}=0.1$ for $u(x, 0)=\sin 2 \pi x$ using $\nu=0.1$.
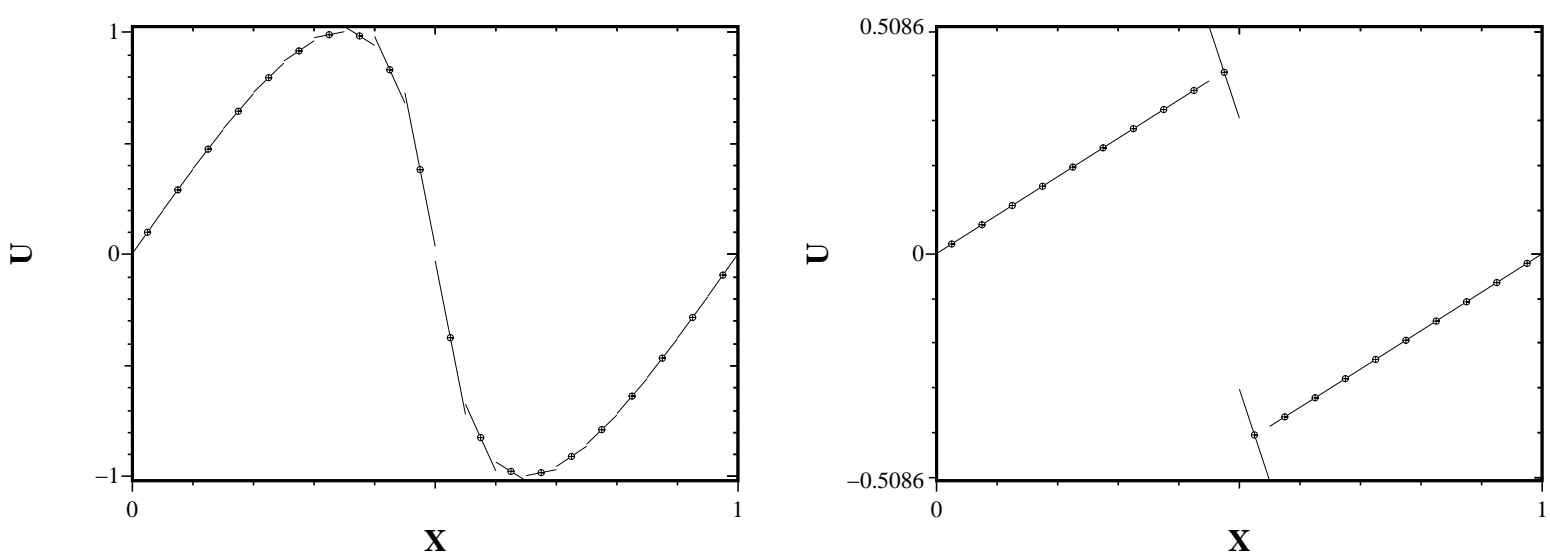

Figure 3: RK2-DG(1) for Burgers' equation with the Godunov flux. The solution is shown at $t=0.1$ when the solution is smooth, and at $t=1.0$ after the shock forms (at $t=1 / 2 \pi$ ) for $u(x, 0)=\sin 2 \pi x$. The reconstruction is plotted and the symbols show the cell mean values. 

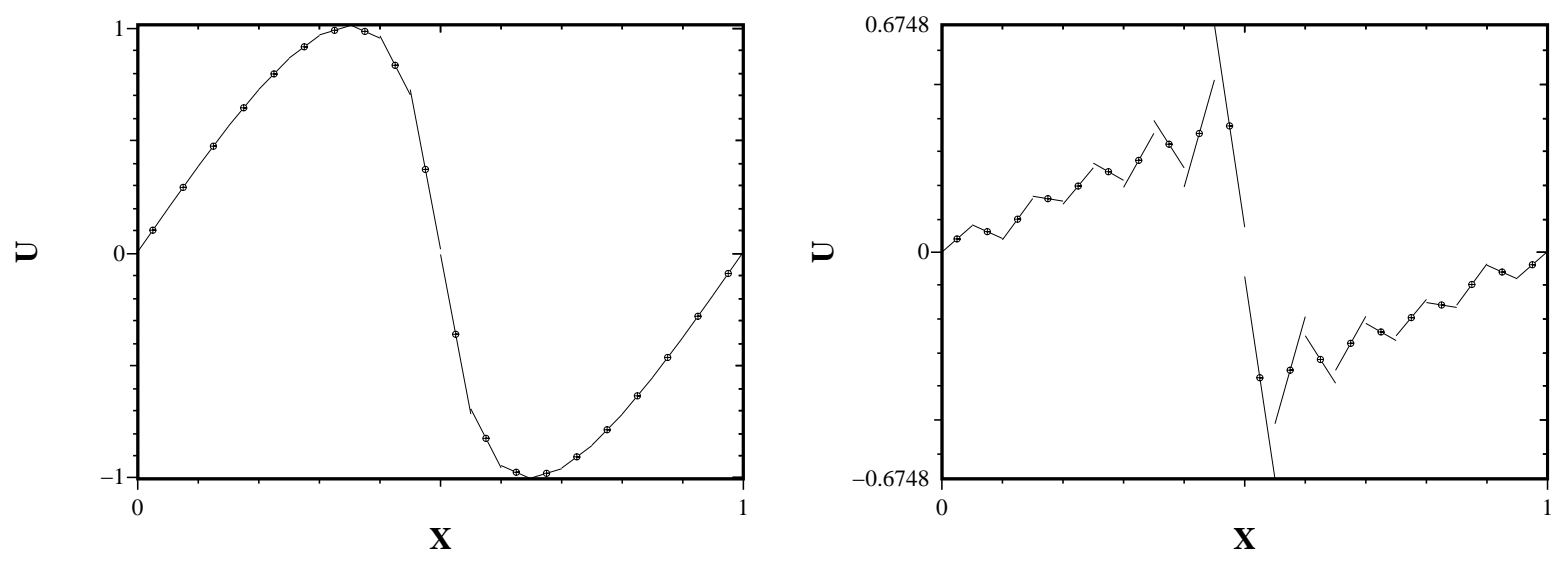

Figure 4: RK2-DG(1) with mLxF flux for Burgers' equation.

\section{References}

[1] B. Cockburn and C.-W. Shu. TVB Runge-Kutta local projection discontinuous Galerkin finite-element method for conservation-laws .2. general framework. Mathematics of Computation, 52:411-435, 1989.

[2] B. Cockburn and C.-W. Shu. The Runge-Kutta discontinuous Galerkin method for conservation laws V : Multidimensional systems. Journal of Computational Physics, 141:199$224,1998$.

[3] R. B. Lowrie. Compact Higher Order Numerical Methods for Hyperbolic Conservation Laws. PhD thesis, University of Michigan, 1996.

[4] J. E. Morel, T. A. Waring, and K. Smith. A linear-discontinuous spatial differencing scheme for Sn radiative transfer calculations. Journal of Computational Physics, 128:445$462,1996$.

[5] W. H. Reed and T. R. Hill. Triangular mesh methods for the neutron transport equation. Technical Report LA-UR-73-479, Los Alamos National Laboratory, 1973.

[6] C.-W. Shu. Total-variation-diminishing time discretizations. SIAM Journal on Scientific and Statistical Computing, 9:1073-1084, 1988.

[7] E. F. Toro. Riemann Solvers and Numerical Methods for Fluid Dynamics. SpringerVerlag, 1997.

[8] B. van Leer. Towards the ultimate conservative difference scheme. IV. A new approach to numerical convection. Journal of Computational Physics, 23:276-299, 1977. 